\title{
Pathogenesis of Pseudotumor (IIH) and Monroe-Kellie Doctrine- Mini-Review
}

ISSN: 2639-0531

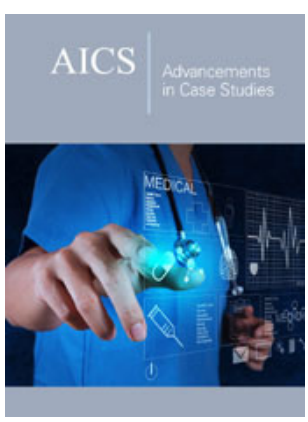

*Corresponding author: Forshing Lui, California Northstate University, College of Medicine: 9700 West Taron Drive, Elk Grove, California, USA

Submission: 酶 August 16, 2021

Published: 海 September 07, 2021

Volume 3 - Issue 2

How to cite this article: Forshing Lui, Shahnawaz Karim, Melanie Chan, Stella Knowlton. Pathogenesis of Pseudotumor (IIH) and Monroe-Kellie Doctrine- MiniReview. Adv Case Stud. 3(2). AICS.000557. 2021.

DOI: 10.31031/AICS.2021.03.000557

Copyright@ Forshing Lui, This article is distributed under the terms of the Creative Commons Attribution 4.0 International License, which permits unrestricted use and redistribution provided that the original author and source are credited.

\author{
Forshing Lui ${ }^{1 *}$, Shahnawaz Karim² ${ }^{2}$, Melanie Chan ${ }^{1}$ and Stella Knowlton ${ }^{1}$ \\ ${ }^{1}$ California Northstate University College of Medicine, USA \\ ${ }^{2}$ Kaiser Permanente Medical Center, South Sacramento, USA
}

\begin{abstract}
Idiopathic Intracranial Hypertension (IIH) is a neurological condition characterized by increased intracranial pressure without any underlying intracranial pathology such as mass lesions in the brain or hydrocephalus. According to the Monroe-Kellie doctrine, there are three main components inside our rigid cranium, brain, blood, and CSF. Increase in volume of one component needs to be compensated by decrease in another component and/or increase in intracranial pressure. The most popular belief about the pathogenesis of IIH is that it is caused by venous sinus stenosis resulting in intracranial venous congestion and increase in ICP. With the recent discovery of another intracranial component, the glymphatic, the Monroe-Kellie doctrine needs to be revisited. In this review, we are providing evidence to define the relative importance of venous sinus stenosis and dysfunction of the glymphatic as the main pathogenetic mechanism for IIH. We also conclude that dysfunction of the glymphatic system is the main pathogenetic mechanism of IIH.
\end{abstract}

Keywords: Idiopathic intracranial hypertension (pseudotumor), Monroe-Kellie doctrine, Venous sinus stenosis, Cerebrospinal fluid, Interstitial fluid, Glymphatic

Abbreviations: IIH: Idiopathic Intracranial Hypertension; CSF: Cerebrospinal Fluid; ICP: Intracranial Pressure; ISF: Brain Interstitial Fluid; VR Space: Virchow-Robin Space

\section{Introduction}

The Monroe-Kellie doctrine provides the basic framework and understanding of intracranial volume and pressure in human beings. According to the doctrine, the three main components are brain, blood, and cerebrospinal fluid (CSF). Any increase in volume of one component will result in decrease in volume of the other components and increase in intracranial pressure (ICP) or both. Idiopathic intracranial hypertension (IIH), also called pseudotumor is a condition characterized by increase in ICP without apparent increase in brain, blood, or CSF volume. The main hypothesized cause is venous sinus stenosis resulting in increased intracranial blood volume and pressure. The recent discovery of the "glymphatics" system consisting mainly of cerebrospinal fluid (CSF) and brain interstitial fluid (ISF) may provide an alternative and better explanation for the pathogenesis of IIH.

\section{Pseudotumor/Idiopathic Intracranial Hypertension (IIH)}

Idiopathic intracranial hypertension, commonly called pseudotumor is a neurological condition characterized by increased intracranial pressure without any underlying intracranial pathology such as mass lesions in the brain or hydrocephalus [1-4]. As of current day, the cause of IIH remains unclear. The typical patients are overweight young females and common clinical presentations include headache with papilledema found upon physical 
exam. Additionally, some medications may increase the risk of developing IIH, including excessive intake of vitamin A and outdated tetracyclines. Neuroimaging studies including CT and MRI remain normal in these patients, with normal or small lateral ventricles. Ultimately, IIH diagnosis is made by high opening pressures on spinal tap with normal CSF constituents and no causes found on neuroimaging including venography to rule out Dural venous sinus thrombosis. The most dreaded complication is visual loss secondary to impaired axoplasmic flow associated with persistent elevation of intracranial pressure (ICP).

At present, the underlying pathogenesis of IIH remains unclear. Altered CSF dynamics, underlying prothrombotic states, female sex hormones, and obesity have all been suggested to play a role2, with recent weight gain in obese patients being the most significant association [5]. The particular mechanism of obesity causing increased ICP is still unclear. The possible mechanisms include increased ICP secondary to increased intrathoracic and intraabdominal pressure because of abdominal obesity [6]. More recently, obesity related inflammation with increased proinflammatory cytokines such as leptin and interleukin- 6 has been implicated [7].

\section{Monroe-Kellie doctrine}

Following closure of fontanelles, our skull forms a rigid cranium with constant volume and content, the latter of which stays constant according to the Monroe-Kellie Doctrine. There are three main components - brain, blood, and CSF inside the cranium, where any increase in volume of one component will either result in decrease in volume of the other components, increase in the intracranial pressure (ICP), or both. When there is an increase in brain volume due to mass lesions such as a tumor or hematoma, the ventricular system will be compressed. When there is excessive volume of CSF in hydrocephalus, the vascular component should decrease. In either scenario, the ICP will be elevated. As such, when there is an increase in intracranial blood volume due to cerebral venous sinus thrombosis or stenosis, the ventricular volume will decrease while the ICP will be elevated. Many recent case reports have found the presence of venous sinus stenoses particularly involving the transverse sinus [8-11], with some studies showing the efficacy of stenting the stenotic transverse sinus in lowering the ICP. Similarly, Dural sinus thrombosis causes increased ICP and may be the cause of pseudotumor cerebri or idiopathic intracranial hypertension (IIH).

However, most known cases of IIH are not associated with Dural sinus thrombosis. In addition to blood and CSF, Iliff et al. [12] demonstrated a brain fluid flow system similar to systemic lymphatics in rodents. They created the term "glymphatics" to describe the flow of cerebrospinal fluid (CSF) from the perivascular space (VR space) accompanying the small cortical arteries into the brain interstitial fluid (ISF). The CSF-ISF flows centripetally towards the perivenous spaces of the deep cerebral venous system, thus draining solutes and wastes in the perivenous spaces out of the system into either the deep cervical lymphatics or large draining veins leaving the skull $[13,14]$. Thus, the glymphatic system exists as the 4th component inside the cranium and that glymphatic outflow obstruction may provide a better explanation for the pathophysiology of IIH.

\section{Venous sinus stenosis as a cause of IIH}

With Monroe-Kellie Doctrine in mind, it is logical to consider increased vascular volume/pressure as an important cause of IIH. Cerebral venous sinus thrombosis has been found to be the cause in many patients with presumed IIH [15]. Most clinical neurologists tend to rule out Dural venous sinus thrombosis when they encounter any patient with clinical diagnosis of IIH. With no evidence of venous sinus thrombosis associated with IIH, increased venous pressure secondary to venous sinus stenosis became the most widely accepted pathophysiological mechanism [16-19]. Stenting of the stenotic transverse sinus demonstrated efficacy in lowering the ICP in patients with IIH has been demonstrated in multiple reports and small case series, as discussed here.

Durst et al. [20] performed consecutive CT angiography on a series of 355 patients without the diagnosis of increased ICP. They found the prevalence of $33 \%$ of unilateral transverse sinus stenosis and 5\% bilateral transverse sinus thrombosis. Lee et al. [21] reported a case of IIH with transverse sinus "obstruction" which partially resolved when the ICP was lowered to $15 \mathrm{~cm} \mathrm{H}_{2} \mathrm{O}$ and completely and immediately resolved when the ICP was reduced by lumbar puncture to $<10 \mathrm{~cm} \mathrm{H}_{2} \mathrm{O}$ [21]. This case showed that the transverse sinus "obstruction" is the result of the raised ICP than the cause of the IIH. Rohr A et al. [22] reported 3 cases in which diagnostic imaging before and after CSF diversion provided evidence that narrowing of the transverse sinuses is a secondary phenomenon, with stent angioplasty of the venous sinuses not to be considered as an appropriate therapeutic approach [22]. In 2017, Buell TJ et al. [23] reported a case of transient resolution of venous sinus stenosis after high-volume lumbar puncture in a patient with IIH. This patient provided strong evidence that the venous sinus stenosis is a downstream effect of the increased ICP rather than its cause [23]. It is clear from these studies that cerebral venous sinus stenting may lower the intracranial pressure in IIH. However, the existing evidence does not definitely support venous sinus (transverse or sigmoid sinus) stenosis as the cause of IIH rather the downstream effect of the raised ICP.

\section{IIH: A consequence of glymphatic outflow obstruction}

Lenck S et al. [24] hypothesized that IIH is related to congestion of the glymphatic system associated with overflow of the glymphatic outflow pathway. Their hypothesis subsequently resulted in more 
debates and disputes. To this end, we analyze this hypothesis with evidence and consequent views. There is approximately $150 \mathrm{~mL}$ of CSF in the average adult, and 24-hour turnover is about $500 \mathrm{~mL}$. Considering the known function of the CSF to be providing buoyancy for the brain, it does not require such a rapid turn-over in volume. There should be more important functions to explain this high CSF turnover. The most logical reason points to metabolic supply and waste clearance for the metabolically active brain. While the traditional view of CSF formation and circulation surrounds choroid plexus production with ventricular circulation and subarachnoid granulation reabsorption, this view has recently been challenged both in domains of CSF production and CSF removal $[25,26]$. In regard to the former, recent evidence supports that, in addition to the choroid plexus, a large volume of CSF is produced directly from the capillaries in the brain secondary to hydrostatic pressure. In regard to the latter, the removal of CSF solely by the arachnoid granulations have been under stronger scrutiny by many studies within the past decade. Tracer studies of CSF flow [27-30] showed drainage through the lymphatics of the cribriform plate, cranial and spinal nerve sheaths. Currently, the most significant contribution to our modern understanding of CSF drainage and brain waste clearance, and existence of a brain structure similar to systemic lymphatics is the concept of "glymphatics" by Iliff $[12,14]$.

By using fluorescent CSF tracers in the rodent model, Iliff et al. [13] were able to demonstrate a large volume of CSF in the subarachnoid space rapidly enters the brain parenchyma along the perivascular spaces (Virchow-Robin space) of the cortical penetrating arteries [13]. Here, the CSF mixes with the brain interstitial fluid (ISF). This CSF influx spreads diffusely and uniformly throughout all cortical deep penetrating arteries centripetally towards the perivenous spaces of the deep draining veins with drainage preferentially restricted towards several larger draining veins. Fluorescent tracer from deep nuclear structures (e.g. striatum and thalami) are then observed to drain medially to the perivenous spaces great vein of Galen and internal cerebral veins, ending up in the deep cervical lymphatics. More recent studies [2730] utilizing MRI with gadobutrol CSF tracer have demonstrated the glymphatic system in humans quite convincingly. Similar to the rodent model, the intrathecally-administered CSF tracer enters the brain from cortical surfaces alongside the periarterial spaces and moves centripetally towards the deep structures.

\section{Relative importance of venous sinus thrombosis and glymphatic clearance in IIH}

With a rigid cranium, we understand that the increased ICP in IIH must be secondary to the increase in volume of one of the intracranial compartments i.e., the brain, blood, CSF, or glymphatics. There is no increase in brain cellular volume nor in CSF ventricular volume, with the latter even reduced in volume due to smaller lateral ventricles in imaging of patients with IIH. The intracranial vascular compartment may be expanded if venous sinus stenosis is the confirmed cause of IIH, yet this is not the case from studies and cases we described above. In addition, the cerebral venous sinuse is a low-pressure system which is compressible. Venous sinus stenosis is commonly apparent in normal patients without increase in ICP [20], and venous sinus stenosis is reversible after ICP lowering with CSF drainage or lumbar puncture [22,23]. On the other hand, the glymphatics system is a fluid filled system with high convective flow volume. Any small change in the volume of this clearance system will result in large change in intracranial pressure. Akins [31] used the term glymphedema to explain the various pathologies related to this glymphatic clearance system. IIH is most likely the result of dysfunction of the glymphatic clearance system, the fourth component within our cranial cavity.

\section{Conclusion}

In summary, available evidence including imaging finding shows that transverse/sigmoid sinus stenosis in patients with IIH is more likely the consequence rather than the cause. Cerebral venous sinus stenosis is less likely the pathogenetic mechanism or cause of IIH. The elevation of ICP in IIH can be better explained by the result of increased volume of CSF-ISF (glymphatic fluid) inside the brain parenchyma, mostly probably the consequence of impaired glymphatic outflow clearance.

\section{References}

1. Wall M (2017) Update on idiopathic intracranial hypertension. Neurol Clin 35(1): 45-57.

2. Ball AK, Clarke CE (2006) Idiopathic intracranial hypertension. Lancet Neurol 5(5): 433-442.

3. Dhungana S, Sharrack B, Woodroofe N (2010) Idiopathic intracranial hypertension. Acta Neurol Scand 121(2): 71-82.

4. Galvin JA, Van Stavern GP (2004) Clinical characterization of idiopathic intracranial hypertension at Detroit medical centre. J Neurol Sci 223 157-160.

5. Rowe FJ, Sarkies NJ (1999) The relationship between obesity and idiopathic intracranial hypertension. Int J Obes Relat Metab Disord 23(1): 54-59.

6. Sugerman HJ, DeMaria EJ, Felton WL, Nakatsuka M, Sismanis A (1997) Increased intra-abdominal pressure and cardiac filling pressures in obesity-associated pseudotumor cerebri. Neurology 49(2): 507-511.

7. Sinclair AJ, Ball AK, Burdon MA, Clarke CE, Stewart PM, et al. (2008) Exploring the pathogenesis of IIH: an inflammatory perspective. J Neuroimmunol 201-202: 212-220.

8. Higgins JN, Tipper G, Varley M, Pickard JD (2005) Transverse sinus stenoses in benign intracranial hypertension demonstrated on CT venography. Br J Neurosurg 19(2): 137-140.

9. Higgins JN, Cousins C, Owler BK, Sarkies N, Pickard JD (2003) Idiopathic intracranial hypertension: 12 cases treated by venous sinus stenting. J Neurol Neurosurg Psychiatry 74(12): 1662-1666.

10. Farb RI, Vanek I, Scott JN, Mikulis DJ, Willinsky RA, et al. (2003) Idiopathic intracranial hypertension: the prevalence and morphology of sinovenous stenosis. Neurology 60(9): 1418-1424.

11. Fera F, Bono F, Messina D, Gallo O, Lanza PL, et al. (2005) Comparison of different MR venography techniques for detecting transverse sinus 
stenosis in idiopathic intracranial hypertension. J Neurol 252(9): 10211025 .

12. Iliff JJ, Nedergaard M (2013) Is there a cerebral lymphatic system? Stroke 44(6 suppl 1): S93-S95.

13. Iliff JJ, Wang M, Liao Y, Plogg BA, Peng W, et al. (2012) A paravascular pathway facilitates CSF flow through the brain parenchyma and the clearance of interstitial solutes, including amyloid $\beta$. Sci Transl Med 4(147): 147ra111.

14. Iliff J, Simon M (2019) The glymphatic system supports convective exchange of cerebrospinal fluid and brain interstitial fluid that is mediated by perivascular aquaporin-4. J Physiol 597(17): 4417-4419.

15. Albert Lin, Rod Foroozan, Danesh-Meyer HV, DeSalvo G, Savino PJ, et al. (2006) Occurrence of cerebral venous sinus thrombosis in patients with presumed idiopathic intracranial hypertension. Ophthalmology 13(12): 2281-2284.

16. Arac A, Lee M, Steinberg GK, Marcellus M, Marks MP (2009) Efficacy of endovascular stenting in Dural venous sinus stenosis for the treatment of idiopathic intracranial hypertension. Neurosurg Focus 27(5): E14.

17. Lisa BE Shields, Christopher B Shields, Yao TL, Plato BM, Zhang YP, et al. (2019) Endovascular treatment for venous sinus stenosis in idiopathic intracranial hypertension: An observational study of clinical indications, surgical technique, and long-term outcomes. World Neurosurg 121: e165-e171.

18. De Simone R, Ranieri A, Bonavita V (2010) Advancement in idiopathic intracranial hypertension pathogenesis: focus on sinus venous stenosis. Neurological Sciences 31(1): 33-39.

19. Dinkin MJ, Patsalides A (2017) Venous sinus stenting in idiopathic intracranial hypertension: Results of a prospective trial. J Neuroophthalmol 37(2): 113-121.

20. Durst CR, Ornan DA, Reardon MA, Mehndiratta P, Mukherjee S, et al. (2016) Prevalence of Dural venous sinus stenosis and hypoplasia in a generalized population. J Neurointerv Surg 8(11): 1173-1177.

21. Lee SW, Gates P, Morris P, Whan A, Riddington L (2009) Idiopathic intracranial hypertension; immediate resolution of venous sinus "obstruction" after reducing cerebrospinal fluid pressure to $<10 \mathrm{~cm} \mathrm{H}_{2} \mathrm{O}$. Journal of Clinical Neuroscience 16(12): 1690-1692.

22. Rohr A, Dorner L, Stingele R, Buhl R, Alfke K, et al. (2007) Reversibility of venous sinus obstruction in idiopathic intracranial hypertension. AJNR Am J Neuroradiol 28(4): 656-659.

23. Buell TJ, Raper DM, Pomeraniec IJ, Ding D, Chen CJ, et al. (2017) Transient resolution of venous sinus stenosis after high-volume lumbar puncture in a patient with idiopathic intracranial hypertension. J Neurosurg 129(1): 153-156.

24. Lenck S, Radovanovic I, Nicholson P, Hodaie M, Krings T, et al. (2018) Idiopathic intracranial hypertension: the veno glymphatic connections. Neurology 91(11):515-522.

25. Oreskovic D, Klarica M (2010) The formation of cerebrospinal fluid: nearly a hundred years of interpretations and misinterpretations. Brain Res Rev 64(2): 241-262.

26. Nakada T, Kwee IL (2019) Fluid dynamics inside the brain barrier: current concept of interstitial flow, glymphatic flow, and cerebrospinal fluid circulation in the brain. Neuroscientist 25(2): 155-166.

27. Davoodi-Bojd E, Ding G, Zhang L, Li Q, Li L, et al. (2019) Modeling glymphatic system of the brain using MRI. Neuroimage 188: 616-627.

28. Ringstad G, Valnes LM, Dale AM, Pripp AH, Vatnehol SA, et al. (2018) Brain-wide glymphatic enhancement and clearance in humans assessed with MRI. JCI insight 3(13): e121537.

29. Watts R, Steinklein JM, Waldman L, Zhou X, Filippi CG (2019) Measuring glymphatic flow in man using quantitative contrast-enhanced MRI. AJNR Am J Neuroradiol 40(4): 648-651.

30. Eide PK, Vatnehol SA, Emblem KE, Ringstad G (2018) Magnetic resonance imaging provides evidence of glymphatic drainage from human brain to cervical lymph nodes. Scientific reports 8(1): 1-10.

31. Akins PT, Guppy KH (2021) Does impaired glymphatic drainage cause glymphedema? A review tailored to neurocritical care and neurosurgery. Neurocrit Care. 